\title{
UNA HISTORIA \\ DE LAS RELACIONES LABORALES \\ PARA NO HISTORIADORES EN UN CONTEXTO DE CRISIS
}

\author{
Agustín Galán García \\ Historia e Instituciones Económicas. Facultad Ciencias del Trabajo. \\ Universidad de Huelva \\ Susana Martínez Rodríguez \\ Historia e Instituciones Económicas. Facultad Ciencias del Trabajo. \\ Universidad de Murcia
}

DOI: $10.1387 /$ lan-harremanak.15441

\section{ABSTRACT}

La mayoría de las universidades españolas han concluido ya el proceso de implantación de los nuevos grados. En unos casos, este proceso ha supuesto, además de una mayor carga de trabajo para los docentes, un mero cambio nominal con escasas manifestaciones prácticas. En otros, por el contrario, las modificaciones han sido más profundas y de mayor aplicación. Incluso tenemos numerosas publicaciones que a modo de balance van ofreciendo los resultados alcanzados y las percepciones obtenidas (Iglesias de Ussel y otros, 2009).

No tenemos constancia, sin embargo, de que los contenidos de las distintas asignaturas se hayan adaptado a la nueva orientación que el grado que nos ocupa ha querido incorporar. Proponemos en esta comunicación una revisión de los programas que se están impartiendo en las asignaturas de historia de las relaciones laborales, al margen de la denominación concreta que reciba en cada una de las universidades. En segundo lugar presentaremos una propuesta de conjunto de cuáles, a nuestro juicio, 
deben ser los contenidos y las orientaciones que debe incorporar un programa que no tiene como destinatarios a futuros historiadores pero que se ha revelado esencial para contextualizar y comprender el carácter realmente complejo y dinámico de las relaciones laborales. En cuarto y último lugar abordaremos el papel explicativo de nuestra materia en el contexto de crisis en el que nos encontramos y nuestra participación, en calidad de docentes de esta materia, en el debate social del momento.

En definitiva, reivindicamos en esta comunicación una actualización de los contenidos atendiendo, al menos, a dos cuestiones que deberian de haberlos condicionado: la llegada el Espacio Europeo de Educación superior y, en segundo lugar, al contexto social y económico en el que nos encontramos.

Palabras clave: Historia de las relaciones laborales, guia docente, contenidos didácticos, función social.

Most Spanish universities have already completed the process of implementation of the new higher education degrees.

In some cases, this process has involved a significant increase in the professors' teaching duties, but a mere nominal change in the name of the subjects. In those cases, the contents of the subjects have not adapted to the new methodological orientation that the degree required.

The aim of this paper is to review the syllabus of History of Economic Labor Relations available in the Spanish (Public) Universities. Secondly, we present a proposal according to our criteria to provide an essential understanding of the complex and dynamic nature of labor relations. Finally, we analyze the explanatory role of the History of Labor Relations in the current crisis context and the University role in this crucial moment.

Key Words: History of Labor Relations, new higher education degrees, syllabus. 


\section{Situación del Grado en la universidad española}

El Grado en Relaciones Laborales y Recursos Humanos, que empezó a rodar en el curso 2008-2009, procede de la fusión de la Diplomatura de Relaciones Laborales y de la Licenciatura en Ciencias del Trabajo (Galán García y Herráiz Martín, 2005). Durante el proceso de elaboración de los nuevos planes de estudio que darían paso a los Grados planteados por el Espacio Europeo de Educación Superior, desde la Asociación Estatal de Centros Universitarios de Relaciones Laborales y Recursos Humanos, se pusieron de manifiesto algunas disfunciones que se estaban produciendo en el proceso y ante las cuales ninguna de las autoridades educativas pertinentes intervino (Galán García, 2011). Apenas ha salido la primera promoción de los «nuevos grados" y el nivel de frustración, al menos en la parte docente, ha sido evidente. Las reformas fueron claramente insuficientes y parciales. La falta de recursos para su aplicación, el exceso de trabajo, la complejidad organizativa que hemos incorporado en el nuevo sistema, y, a nuestro juicio, la falta de adaptación de los contenidos docentes pueden estar en la raíz de aquella sensación.

En el actual grado está presente la materia de Historia, tal y como quedó recogido en el Libro Blanco, en el bloque temático denominado "Historia social $y$ de las relaciones laborales» (Lerma Montero, 2005). El objetivo de la comunicación es avanzar en la elaboración de un programa de la asignatura que pueda servir de referencia en el panorama nacional. Proponemos en esta comunicación una revisión de los programas que se están impartiendo en las asignaturas de historia de las relaciones laborales, al margen de la denominación concreta que reciba en cada una de las universidades. En segundo lugar presentaremos una propuesta de conjunto de cuáles, a nuestro juicio, deben ser los contenidos y las orientaciones que debe incorporar un programa que no tiene como destinatarios a futuros historiadores pero que se ha revelado esencial para contextualizar y comprender el carácter realmente complejo y dinámico de las relaciones laborales. En tercer y último lugar abordaremos el papel explicativo de nuestra materia en el contexto de crisis en el que nos encontramos y nuestra participación en el debate social del momento.

Uno de los resultados descriptivos de la comunicación es una primera revisión de algunos de los programas de la asignatura Historia de las Relaciones La- 
borales. La muestra utilizada deriva de la web de la Asociación Española de Historia Económica. De las 55 universidades que han participado en la web (http:// www.aehe.net/docencia-seccion/guias-docentes.html) existen 19 casos que han puesto a disposición de los internautas las guías docentes de las asignaturas de Historia de las Relaciones Laborales o vinculadas a la Historia Económica y/o Social. No obstante consideramos que la muestra es lo suficientemente interesante para realizar este primer ejercicio y poder ampliarlo en ediciones sucesivas.

\section{Orientaciones, competencias y contenidos de la asignatura de historia}

El papel de una asignatura de historia en el proceso de formación de los futuros graduados en relaciones laborales y recursos humanos consiste en ofrecer al

Tabla 1

Contenidos formativos, destrezas y habilidades

Contenidos formativos mínimos

— Fundamentos sociales, políticos y económicos del mundo contemporáneo.

- Historia de los agentes socioeconómicos en los mercados de trabajo.

— Distribución de la riqueza de las sociedades capitalistas contemporáneas. Las alternativas al sistema liberal-capitalista.

- El papel de las instituciones en la configuración de las relaciones laborales.

— Evolución de la formas de trabajo.

—Evolución histórica de las políticas sociales en el ámbito nacional e internacional.

Capacidades, destrezas, habilidades

- Capacidad para realizar informes orales y escritos sobre aspectos referidos al análisis temporal del trabajo, las relaciones laborales y el funcionamiento de los mercados de trabajo.

- Capacidad para buscar, organizar, gestionar e interpretar informaciones cualitativas y cuantitativas referidas a las relaciones laborales y al funcionamiento de los mercados de trabajo.

- Capacidad para contextualizar, comparar y analizar críticamente los fenómenos, sucesos y procesos que tienen lugar en la evolución histórica del trabajo, las relaciones laborales y los mercados de trabajo.

— Capacidad para comprender el carácter dinámico y cambiante de las relaciones laborales.

—Adquisición de destrezas para el análisis de realidades sociales complejas pasadas y presentes.

- Dominio de procedimientos, instrumentos y terminología de la ciencia histórica para el análisis socio laboral.

Fuente: Lerma Montero, 2005. 
alumno una visión realista de la interacción de los diferentes agentes y factores que intervienen en la dinámica de las relaciones de trabajo, sabiendo que son relaciones cambiantes, sujetas al contexto social, político y económico en el que se desarrollan. Este debe ser el eje argumental del programa (Galán García, 2011). Al mismo tiempo debe explicar los antecedentes históricos de los grandes ámbitos de actuación profesional en los que tendrá que desarrollar su trabajo; es decir, la gestión de los recursos humanos - ya sea en el interior de una organización o como asesor externo-; el desarrollo, gestión y evaluación de las políticas socio-laborales, fundamentalmente políticas de empleo y de seguridad social, y, finalmente, hacer lo propio en el ámbito de la salud laboral o la prevención de riesgos laborales. De forma sinóptica en la tabla 1 recogemos los contenidos formativos mínimos, las capacidades, destrezas y habilidades de la materia.

\section{Historia de las Relaciones Laborales y materias afines}

La primera nota que destaca es la diversidad de las veintidós materias sobre historia, ocho están estrictamente dedicadas a la Historia de las Relaciones Laborales (H. ${ }^{a}$ RRLL en adelante), siete de contenido general y una específica de la Historia de las Relaciones Laborales en España (U. Huelva). En la Universidad de Zaragoza se imparte una materia denominada Historia Social y de las Relaciones Laborales y en la de Córdoba una de Historia y Teoría de las Relaciones Laborales. Los demás casos de la muestra son asignaturas que tienen un fuerte componente en historia económica, historia social o historia del trabajo. Algún caso más singular, en cuando a denominación sería el de Casos de Historia de la Universidad Pablo de Olavide.

En cuanto al análisis de sus contenidos, lo primero que hemos hecho, ha sido sistematizar los contenidos de las asignaturas que son semejantes y uno de los motivos reside en que los docentes están adscritos al área de Historia Económica, por lo que en realidad imparten un programa de Historia Económica (Mundial Contemporánea) con contenidos específicos de Historia de las Relaciones Laborales o del Mercado Laboral. La tabla 3 sintetiza los programas de las materias Historia de las Relaciones Laborales. Existe una esmerada atención al capitalismo moderno, a los inicios de la industrialización y a la primer parte del siglo Xx. Desde el punto de vista cronológico, los temarios llegan hasta finales del xx, o incluso algunos hasta el mismo año de la presentación de la guía docente. El último tema de la asignatura en la U. de Murcia se titula «El fin del siglo xx: nuevos escenarios sociales y laborales». La U. de Sevilla opta por un título más concreto «Las relaciones laborales en la era dela globalización y el neoliberalismo, 1980-2012». U. Huelva y U. Almería optan por concepciones más generales que no necesariamente llegan hasta el siglo Xxi: «Las relaciones labora- 


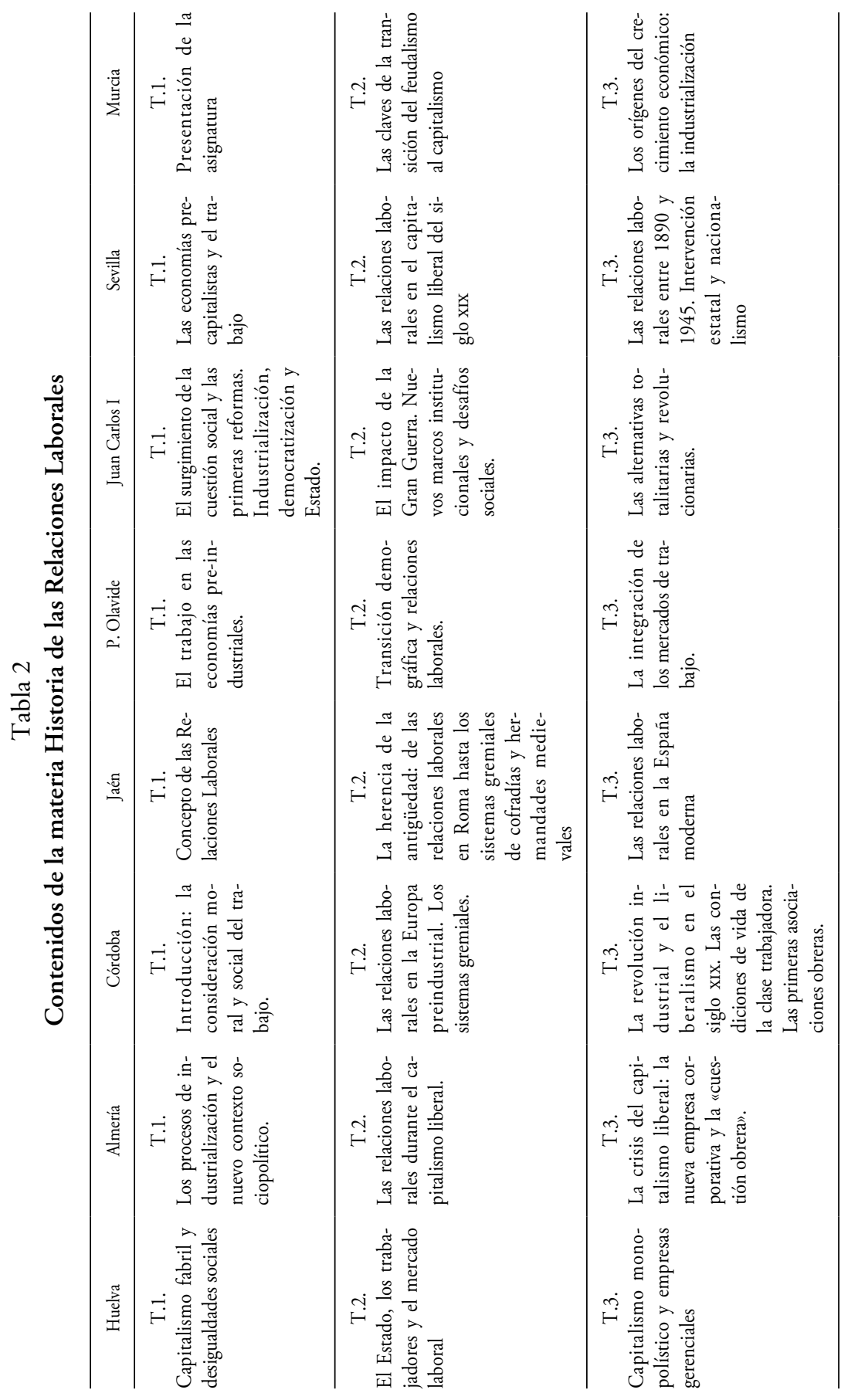




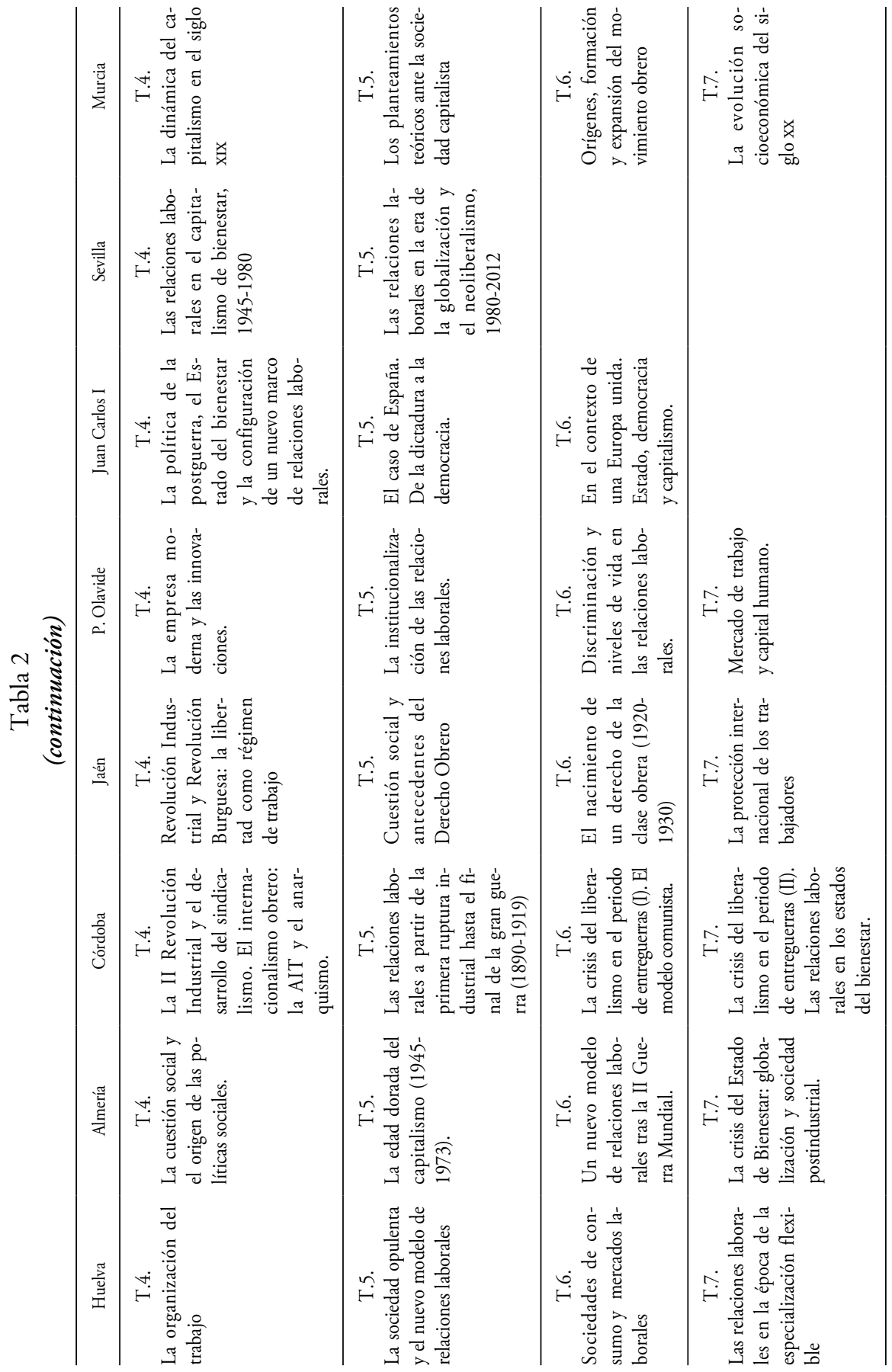




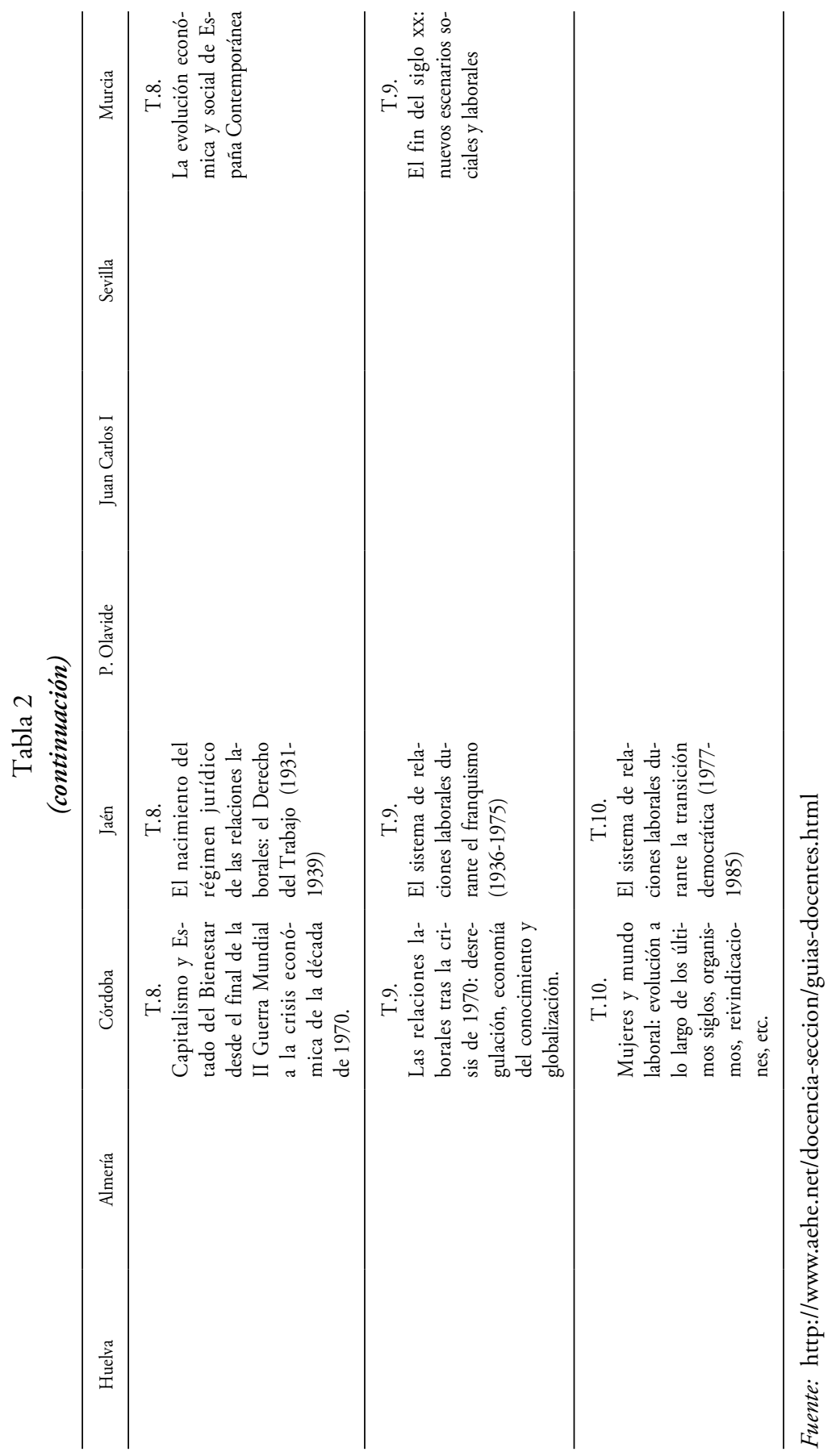


les en la época de la especialización flexible» $\mathrm{y}$ «La crisis del Estado del Bienestar: globalización y sociedad postindustrial».

Esta homogeneidad es debida a que la mayor parte de las asignaturas están impartidas por profesores del área de Historia e Instituciones Económicas, de lo que también se infiere el acusado rasgo de una Historia Económica al uso, centrada en el proceso de industrialización y post-industrialización.

Las propuestas impartidas en la U. de Córdoba y U. de Jaén destacan por la amplitud descriptiva del temario, si bien disponen solo de un cuatrimestre y un total de 150 horas totales de estudio presentan una guía docente con diez temas. La U. de Murcia presenta un temario también largo de nueve temas. Las demás optan por un temario más discreto en cuanto a su extensión.

Siguiendo la misma metodología, en la tabla 3, recogemos los contenidos de las 10 materias que son Historias; su variedad impide que los comentarios sean tan visuales como los de la Tabla 2. En primer lugar existe un grupo de materias que, al igual que el conjunto de Historia de las Relaciones Laborales, reclaman una urgente actualización de contenidos ya que cubren un marco cronológico insuficiente para las necesidades de los futuros graduados, a los que les urge un conocimiento del funcionamiento del mundo sobre todo en los últimos 30 años. Aunque en este bloque de asignaturas el sesgo es más político y social que en la de H. ${ }^{a}$ RRLL creemos que de nuevo el profesorado precisa adaptar y modernizar sus contenidos. Otro rasgo preocupante es la excesiva longitud de los temarios, aunque solo sea formal. 


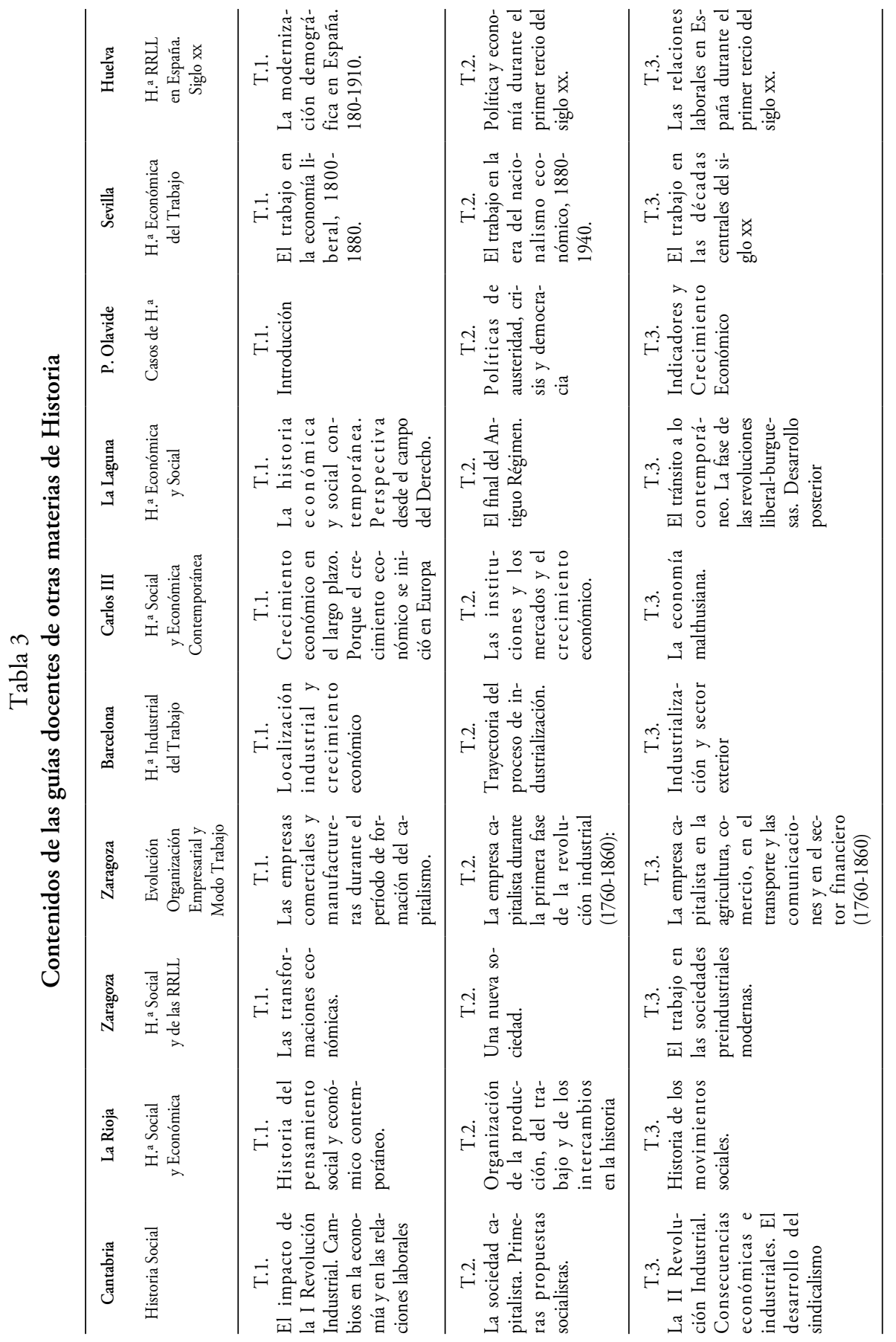




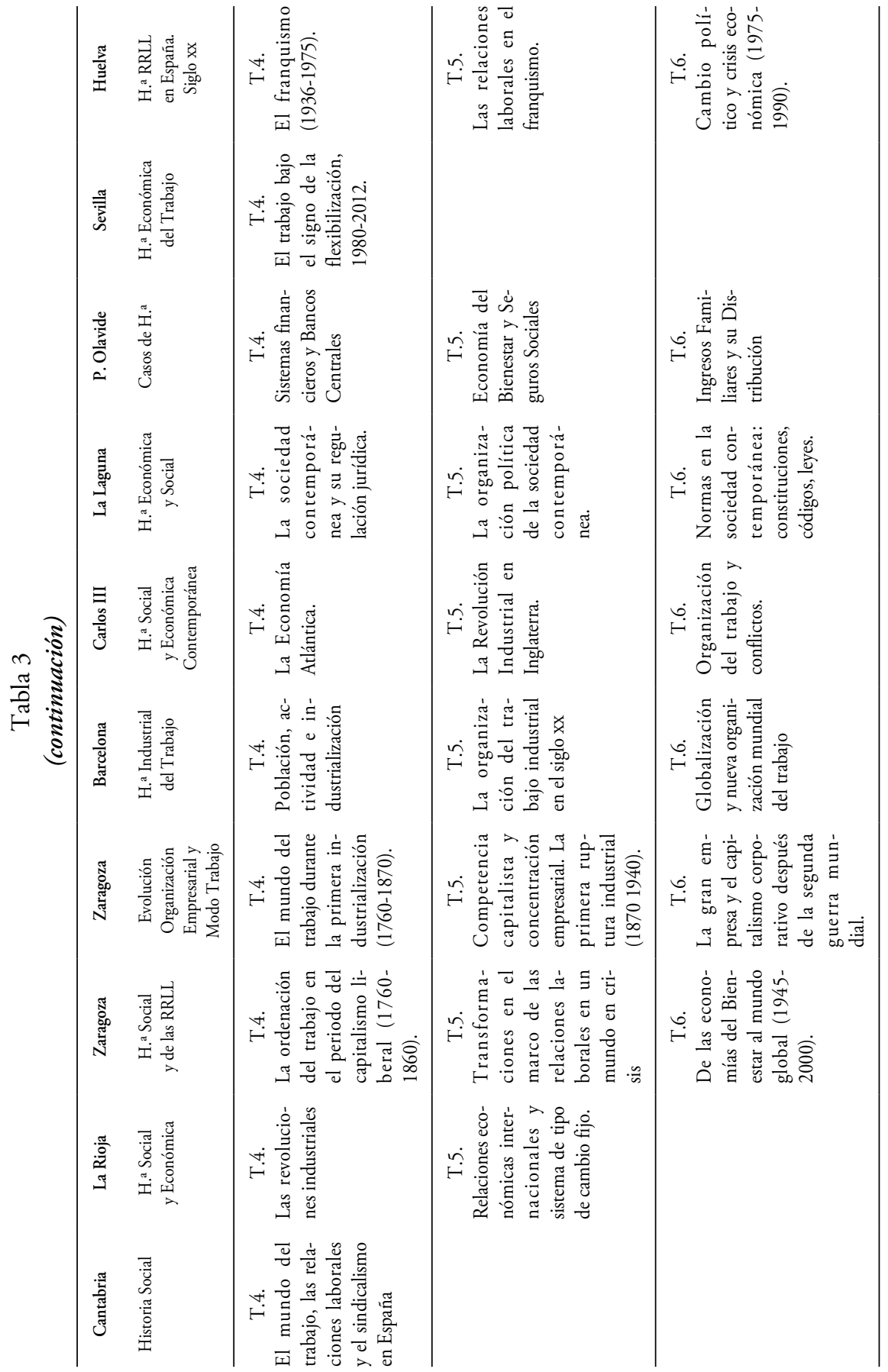




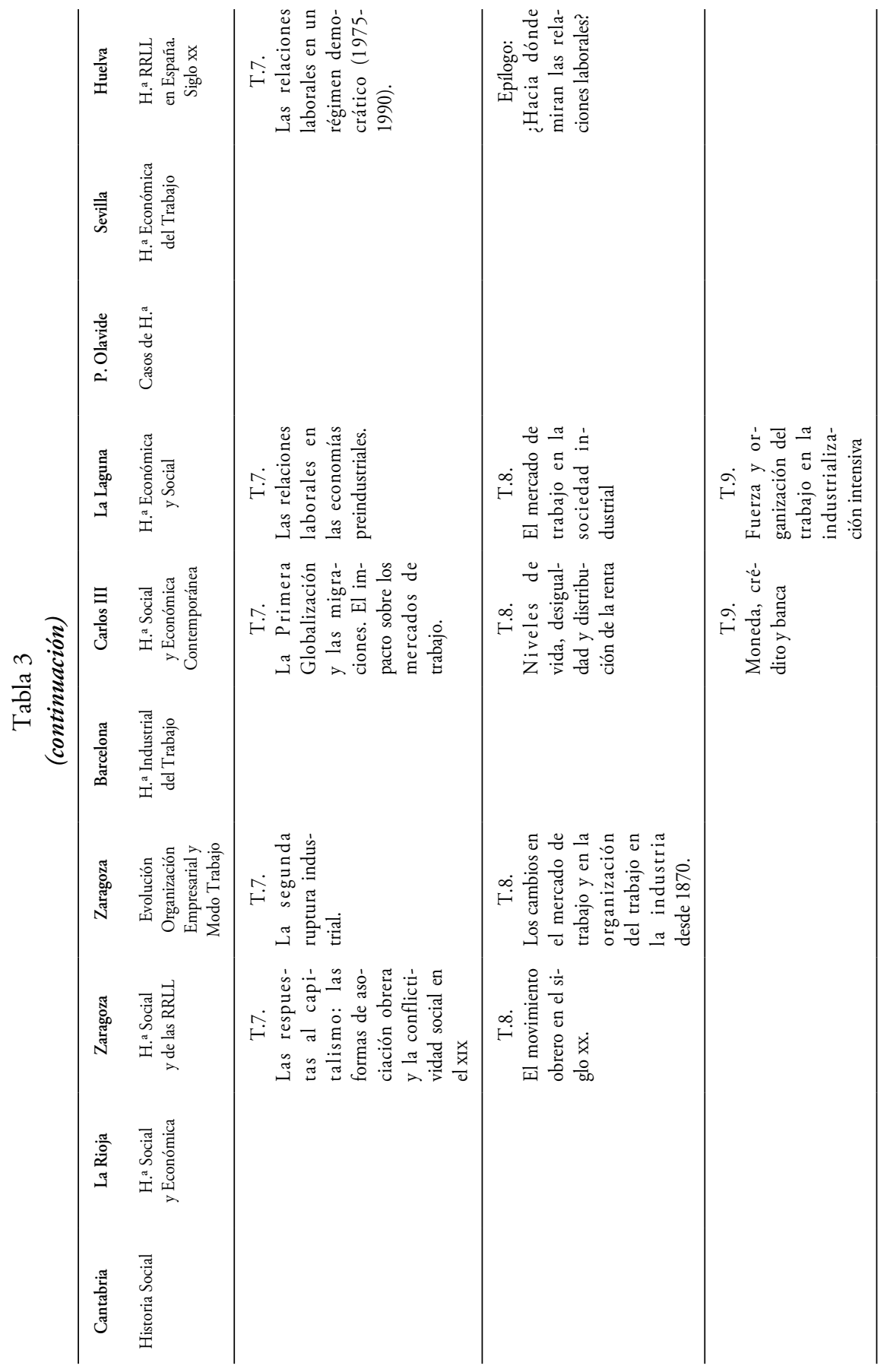




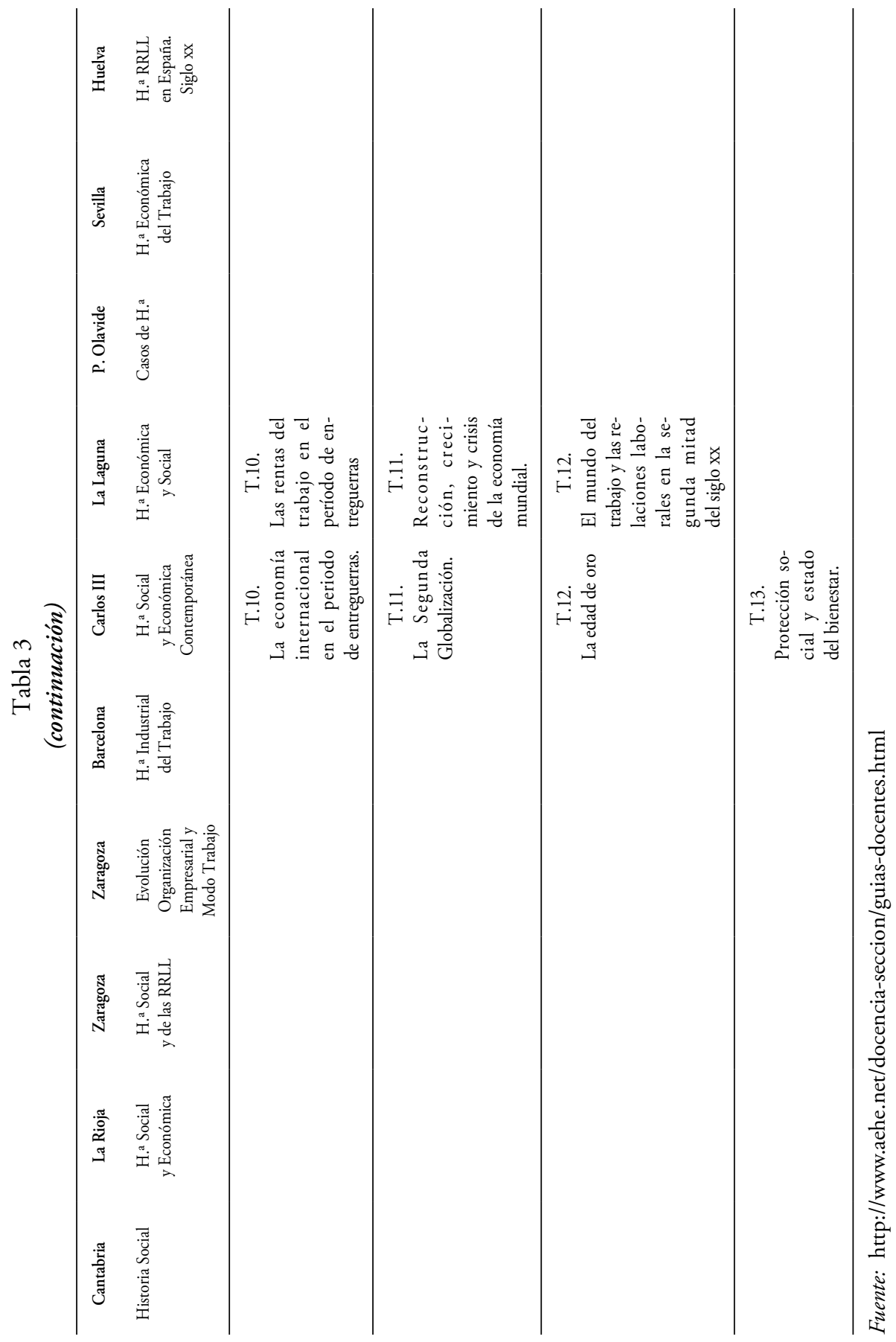




\section{Una propuesta de contenidos para la historia de las relaciones laborales}

La propuesta que realizamos está profundamente influida por nuestra área. Creemos que los cursos de Historia Económica y de Historia de las Relaciones Laborales en particular deben servir para que los alumnos mejoren su capacidad de entender, integrar, asimilar y contextualizas el conjunto de sus estudios en el Grado de Relaciones Laborales y Recursos Humanos. Siguiendo las directrices de Llopis (1991), la asignatura proporciona:

a) Cultura histórica, un elemento fundamental para la contextualización, ubicación y localización de problemas.

b) Aporta unas técnicas de análisis especialmente apropiadas para el examen del largo plazo.

c) Proporciona una metodología especialmente diseñada para el estudio de las interrelaciones entre los factores económicos y los extraeconómicos.

A ninguno se nos escapa que todos nuestros graduados deberán superar entrevistas personales donde debe mostrar habilidades sociales, competencias, técnicas, y por supuesto, un nivel de cultura general. Materias como Historia de las Relaciones Laborales son fundamentales para dotar de mayor madurez intelectual a los estudiantes. Igualmente importante es lograr que los propios estudiantes valoren el capital intelectual que supone la cultura histórica, y más concretamente el método histórico (Llopis, 1991, 29).

Para suscitar el debate y la reflexión proponemos algunos objetivos que consideramos debería incluir un «hipotético programa ideal». Respecto de los objetivos, planteamos tres ideas:

a) Reflexionar sobre los diversos factores que influyen en las relaciones laborales (siglos XIX y sobre todo XX y XXI). Transmitir y dar a conocer a los alumnos las condiciones políticas, sociales y económicas básicas para entender el contexto de los distintos sistemas de relaciones laborales que se suceden en los siglos el mundo contemporáneo, para comprender su carácter dinámico y cambiante.

b) Analizar el papel de los distintos agentes sociales (estado, patronos, trabajadores-organizaciones obreras) que participan en las relaciones laborales del período contemporáneo.

c) Ayudar a contextualizar los contenidos que ofrecen las materias básicas de la titulación; nos referimos a la Economía, el Derecho, fundamentalmente de del Trabajo y de la Seguridad Social, las políticas de empleo, la empresa, gestión de los recursos humanos, etc. La historia debe jugar aquí un papel auxiliar por su duración pero esencial por la visión. Esto 
obliga a una estrecha coordinación o, al menos conocimiento, de lo que imparten el resto de las áreas de conocimiento que participan en la titulación.

El cumplimiento de aquellos objetivos requiere, necesariamente, de la coordinación con el resto de asignaturas. (Lerma Montero, 2005). Aquel ejercicio nos obliga a:

a) estar muy atento al contenido del resto de asignaturas para engarzar sus contenidos con la nuestra, y

b) a ser conscientes de que nuestra materia, en esta titulación es meramente auxiliar pero no por ello menos importante. En este mismo sentido hemos de trabajar en propuestas de coordinación concretas. Aquí sí hemos conocido un avance importante, ya sea por la vía de la coordinación de cursos o de titulación, por la vía de la coordinación informal a través de seminarios, etc., o a través de la organización de actividades conjuntas con otras áreas de conocimiento.

Respecto a los contenidos, definimos tres grandes bloques temáticos ${ }^{1}$ :

a) Los mercados laborales durante el capitalismo liberal. El contexto de las revoluciones industriales y políticas en el siglo XIx. Las características de los mercados laborales y sus escenarios de trabajo en la primera etapa del capitalismo. Las formas de oposición al sistema y la aparición del sindicalismo. Este bloque perdería protagonismo frente a los dos siguientes.

b) El gran capitalismo y las relaciones laborales. Los profundos cambios políticos y sociales hasta la época de entreguerras. La aparición de los sindicatos de clase, la negociación colectiva, las modernas empresas multiunitarias y los seguros sociales.

c) La época del consumo de masas y el modelo de relaciones laborales (19452010). Las bases sociales y económicas del capitalismo en este período. Las modificaciones en el mundo del trabajo y la definición del modelo de relaciones laborales. Los nuevos escenarios laborales y sociales.

A modo de epílogo y tratando de dar una respuesta desde la Historia a la situación de crisis prolongada que venimos padeciendo, es necesario darle presencia a esta cuestión en los programas de la asignaturas.

1 Damos por hecho que todos los colegas que imparten docencia en este grado conocen con cierto detalle las salidas profesionales de los futuros graduados. Si esto no es así todo lo que estamos apuntando aquí carece de sentido, por un lado, y, lo que es más graves, su falta de compromiso los hace incompetentes para la función que nos ocupa, por otro. 
Desde un punto de vista más específico habría que incorporar algunos temas, todos ellos de gran actualidad pero que apenas tienen cabida en los programas vigentes. A modo de propuesta, consideramos que habría que incluir:

1. Siniestralidad laboral. Esta materia como objeto de investigación ha evolucionado de una manera considerable en los últimos años hasta convertirse en una materia especializada. Se hace necesario ofrecer una visión histórica de la misma y coordinarnos con las áreas limítrofes, es decir, Medicina Preventiva y Salud Pública, Derecho del Trabajo y de la Seguridad Social, Derecho de la Seguridad y Salud en el Trabajo, Psicología Social, Economía del trabajo, etc.

2. Políticas de empleo y sistemas de colocación: ¿Podemos considerar como políticas de empleo los repartos de tierras de la segunda mitad del siglo XVIII? Además habría que abordar los orígenes y el desarrollo de los sistemas de colocación, formación y orientación, etc., coordinando contenido con las áreas encargadas de impartir las políticas socio laborales, es decir, Derecho del Trabajo y de la Seguridad social y Economía, fundamentalmente.

3. El peso de la economía social sobre el conjunto de nuestra economía es más que evidente, tanto desde el punto de vista del empleo como de su aportación al PIB. Aquí será necesario trabajar conjuntamente con las áreas de Organización de empresa, Economía y Derecho Mercantil.

4. La Responsabilidad Social Corporativa es otra de las variables temáticas que encuentra en la historia un potencial explicativo único. La alusión a este concepto y la búsqueda de sus precedentes en el pasado es otra línea de trabajo que convendría incorporar. Da la impresión que este es un concepto de última generación. Basta una mirada a su definición, objetivos, difusión, etc. Para entender que no es sino la última evolución que ha conocido la empresa en sus relaciones con el entorno en el que se inserta. A modo de ejemplos y como un primer material de referencia podemos citar los trabajos de Domínguez Martin (2008) o el de Galán García (2012) Además, claro está de los manuales de historia de la empresa que solemos utilizar.

5. Culturas del trabajo. Los valores que rigen las organizaciones son la esencia de las mismas. La cultura que generan explican en gran parte su desarrollo. (Gimbert, 2011,41-61). No obstante su importancia hemos cedido este interesantísimo terreo a antropólogos, psicólogos de las organizaciones y estos, a su vez, se lo discuten con las áreas de organización de empresa.

Parece obvio a estas alturas que, tanto los temas que apuntamos como los programas a los que nos venimos refiriendo, deben venir impregnados por una orientación comparativa especialmente necesaria en estos momentos. Hemos de 
hacernos eco, con las limitaciones cronológicas que tenemos, de lo que ha ido ocurriendo en otras zonas del mundo, antaño aisladas (China, Indias, Australia, África, etc.), pero sin cuya participación hoy día no se entendería la dinámica de los mercados de trabajo. Del mismo modo, se hace obligatorio tratar de introducir temas de actualidad y, al mismo tiempo, tratar de arrastrar hasta nuestros días las líneas argumentales que vertebran los programas. De lo contrario tendremos realmente difícil el captar la atención de nuestros alumnos, porque si no lo hacemos así, nos va a resultar realmente complicado hacerles ver la utilidad de nuestra disciplina.

Esta visión de la asignatura descansa en la adscripción al área de conocimiento dominante (Historia e Instituciones Económicas) pero solivianta los problemas que vemos en los programas actuales: necesidad de actualización y conexión con las áreas de conocimiento fundamentales del grado.

No obstante también es importante realizar un ejercicio de autocrítica que compense la complacencia que pudiera desprenderse de las páginas anteriores.

Sin duda, los docentes de la materia somos los primeros responsables en conseguir que los estudiantes se interesen por ella, de hacerla atractiva (Román Cervantes, 2007). Con independencia de nuestra preferencias es más que evidente que un joven de 20 años puede mostrar un mínimo - por no decir nulo- interés por la Primera Revolución Industrial Británica. Sin embargo, por las circunstancias que está viviendo posiblemente tenga interés en conocer cómo ha sido la incorporación de las mujeres al mercado laboral en la segunda mitad del xx, conocer el origen de la Seguridad Social española y por qué hoy está siendo tan cuestionada, por qué la siniestralidad laboral depende del marco institucional particular y cómo una determinada concepción del trabajo puede tener implicaciones en los valores sociales y morales de la sociedad. En esta propuesta abierta planteamos una actualización de contenidos que nos permita ganar más visibilidad en el grado, interrelacionando contenidos con los de otras materias y poniendo en valor una asignatura más volcada en los problemas contemporáneos, menos eurocentrista y que ponga el valor la capacidad de interrelación que caracteriza a nuestra área (Llopis, 1991, 36).

\section{La función social del docente historiador y nuestra aportación al debate social}

Y, por último, proponemos una revisión del papel social del historiador, en este caso, en su calidad de docente, si es que esta puede vivir aislada de su condición de investigador.

El título preliminar de la Ley Orgánica de Reforma Universitaria (LRU) de 1987, pretendía establecer un nuevo marco para la renovación de la vida aca- 
démica en el que sus protagonistas debían ser los verdaderos motores de aquella aventura pos-transición; «El profesorado y los alumnos tienen, pues la clave de la nueva Universidad que se quiera conseguir, y de nada servirá ninguna Ley si ellos no asumen el proyecto de vida académica que se propone, encaminada a conseguir unos centros universitarios donde arraiguen el pensamiento libre y crítico y la investigación. Sólo así la institución universitaria podrá ser un instrumento eficaz de transformación social, al servicio de la libertad, la igualdad y el progreso social para hacer posible una realización más plena de la dignidad humana.... ${ }^{2}$. El fomento del pensamiento libre y crítico, que es a lo que aquí nos queremos referir, estaba recogido de una manera nítida.

Casi veinte años más tarde, la Ley Orgánica de universidades, decía dibujar el escenario para mejorar la calidad en todas las áreas de la actividad universitaria es básico para formar a los profesionales que la sociedad necesita, desarrollar la investigación, conservar y transmitir la cultura, enriqueciéndola con la aportación creadora de cada generación y, finalmente, constituir una instancia crítica y científica, basada en el mérito y el rigor, que sea un referente para la sociedad española. Así, la Ley crea las condiciones apropiadas para que los agentes de la actividad universitaria, los genuinos protagonistas de la mejora y el cambio, estudiantes, profesores y personal de administración y servicios, impulsen y desarrollen aquellas dinámicas de progreso que promuevan un sistema universitario mejor coordinado, más competitivo y de mayor calidad ${ }^{3}$.

La Ley orgánica de 2007 que venía a reformar la anterior, resumía las funciones de la universidad de una manera muy simple: La creación, desarrollo, transmisión y crítica de la ciencia, de la técnica y de la cultura y la preparación para el ejercicio de actividades profesionales que exijan la aplicación de conocimientos y métodos científicos y para la creación artística ${ }^{4}$.

Es decir, el propio marco regulador de nuestra actividad iba relegando cada vez más la función crítica de la institución en beneficio de la transmisión del conocimiento y de la respuesta obligada a las necesidades del mercado. De la creación de un pensamiento libre y crítico de la primera, a la constitución de una instancia crítica en la segunda, y a la creación, desarrollo, transmisión y crítica de la ciencia. Casi que treinta años después el papel crítico de las universidades debía limitarse al cuestionamiento de lo científico5 .

2 Ley Orgánica 11/1983, de 25 de agosto, de Reforma Universitaria.

3 Ley Orgánica 6/2001, de 21 de diciembre, de Universidades.

4 Ley Orgánica 4/2007, de 12 de abril, por la que se modifica la Ley Orgánica 6/2001, de 21 de diciembre, de Universidades.

5 Y todo, además, debía estar en perfecta consonancia con lo que los poderes establecidos consideraban que debía ser lo politicamente correcto. 
En síntesis, el propio marco institucional, el sistema de reconocimiento de la investigación y el crecimiento económico de la segunda mitad de los noventa hasta mediados de la primera década del siglo XXI, fueron las mejores barreras contra el fomento de la crítica a los poderes establecidos y al propio sistema universitario (Galán García, 2011,15-45). Sin embargo, la crisis económica vino a poner sobre la mesa las contradicciones que se venían produciendo y la colaboración del sistema educativa en su posible explicación. Nos estamos refiriendo a la «Iniciativa internacional de estudiantes para el pluralismo en Economía» ${ }^{6}$. No debemos despreciar, a modo de precedente, el trabajo de F. Noiville (2011), cuyo título es más que revelador de lo que luego vendría: «Soy Economista y os pido disculpas». Sea como fuere, frente a la atonía y el silencio, casi generalizado, de la Academia, han sido los alumnos los que han levantado la voz para exigir un cambio radical en la manera de enseñar la economía.

«No es sólo la economía mundial que está en crisis. La enseñanza de la economía está en crisis también, y esta crisis tiene consecuencias que van más allá de los muros universitarios. Lo que se enseńa en la Universidad moldea las mentes de la próxima generación de políticos, y por lo tanto da forma a las sociedades en que vivimos. Más de 65 asociaciones de estudiantes de economía de más de 30 países diferentes, creen que es el momento de reconsiderar la forma en que la economía se enseña. No estamos satisfechos con la dramática reducción del plan de estudios que se ha producido en el último par de décadas. Esta falta de diversidad intelectual no sólo perjudica la educación y la investigación. Limita nuestra capacidad para lidiar con los desafíos multidimensionales del siglo xxI de la estabilidad financiera, la seguridad alimentaria y el cambio climático. El mundo real debe ser traído de vuelta a las aulas, así como el debate y el pluralismo de teorías y métodos. Este cambio ayudará a renovar la disciplina y en última instancia, crear un espacio en el que las soluciones a los problemas de la sociedad se pueden generar.

Unidas a través de fronteras, pedimos un cambio de rumbo. No pretendemos tener la respuesta perfecta, pero no tenemos ninguna duda de que los estudiantes de economía se beneficiarán de la exposición a los diferentes puntos de vista e ideas. El pluralismo no sólo ayudará a enriquecer la enseñanza y la investigación y revitalizar la disciplina. Más que esto, el pluralismo conlleva la promesa de llevar la economía de nuevo al servicio de la sociedad. Tres formas de pluralismo deben estar en el centro de los planes de estudio: teórica, metodológica e interdisciplinaria... Además, es esencial que los planes de estudio principales incluyan cursos que proporcionan el contexto y fomentan el pensamiento reflexivo sobre la economía y sus métodos en sí, incluyendo la filosofía de la economía y la teoría del conocimiento. También, porque las teorías no pueden ser plenamente entendidas independientemente del contexto histórico en el que se formularon, los estudiantes deben ser expuestos sistemáticamente a la historia del pensamiento económico y de la literatura clásica en la economía, así como a la historia económica. En la actualidad, este tipo de cursos son inexistentes o marginados a los márgenes de los planes de estudios de economía...»

${ }^{6}$ https://www.isipe.net/open-letter (10.V.2014). 
Los alumnos vienen a decir que explicar economía es hacer política. Hacer investigación histórica es una manera de hacer política. Podemos añadir sin temor a equivocarnos que explicar historia, o, enseñar historia, también es una forma de hacer política. En este sentido sintonizamos perfectamente con E. Florescano cuando afirma que «El historiador, y cualquier científico social, debe asumir el significado y la responsabilidad de su función social, una que, al final, es de orden moral... En ese oficio de dar sentido al pasado no se puede alegar inocencia ni neutralidad. Incluso aquel que simplemente se propone historiar sobre la vida de los santos está tomando partido en el presente y ni qué decir de quien aborda directamente los temas del poder» ${ }^{7}$.

Continua el autor mexicano retratando una situación en la que los intereses políticos y gremiales no han estado del todo ausentes. Apenas salvando ligerísimos matices nos debe resultar familiar. «En México ha ocurrido una división del trabajo muy perversa: la clase gobernante ha excluido de su círculo a los académicos, luego, dentro de las universidades, el grupo de los administradores ha excluido a los investigadores, y estos últimos se han dedicado, para defenderse, a cercar y delimitar su espacio de especialización mediante la creación de agrupaciones gremiales que deciden por sí y ante sí qué es lo que vale y lo que no, al punto que hoy el valor del investigador está en función de saber cada vez más sobre muy poco... El resultado ha sido el aislamiento de la actividad del profesional de las fuerzas que hacen la historia y de las necesidades del entorno social en que opera. Se trata de un perfecto círculo vicioso de intereses bien atrincherados que termina por favorecer "historia del gremio para el gremio» («icientos de miles de libros guardados en las bodegas!») y que impide cumplir con la función social de la historia. Y esto no ocurre sólo en México, en diferente medida en muchos otros ámbitos universitarios también se observa "la proliferación de obras que no aportan nada significativo» ${ }^{8}$.

Además, aquella responsabilidad de la que venimos hablando hemos de ejercerla, según las palabras de Hobsbawm (1998, 13), cuando vivimos en una especie de presente permanente en el que la destrucción del pasado, o, más bien, de los mecanismos sociales que vinculan la experiencia contemporánea del individuo con la de generaciones anteriores, se ha vuelto característica. Y esto es precisamente lo que otorga mayor trascendencia a los historiadores, cuya tarea consiste en recordar lo que otros olvidan» (Correa Ramírez, 2011).

¿Pero qué queremos decir con toda esta ristra de consideraciones que podrían parecer faltas de coherencia? El marco regulador de nuestra institución, el sistema de promoción, especialmente el modo de valorar la investigación, el pro-

\footnotetext{
7 http://gustoporlahistoria.com/tag/comentarios-sobre-la-funcion-social-de-la-historia-deenrique-florescano/ (19.05.2014)

8 Ibidem.
} 
pio crecimiento económico de los últimos años, nos han llevado al abandono de nuestra responsabilidad social como docentes comprometidos con el papel social que debe jugar el conocimiento de la historia. $\mathrm{O}$, al menos, no hemos sido capaces de trasladarla más allá de nuestros programas; casi me atrevería a decir más allá de nuestros despachos. Ni siquiera la ayuda de las redes sociales hemos sabido aprovecharlas.

Los alumnos de económicas nos han lanzado un guante que no hemos sabido recoger. Hacen alusión directa, expresa, a la necesidad de recurrir a nuestra disciplina para entender el devenir y el presente económico. No podemos, no debemos dejar pasar esta oportunidad. Propongamos, dinamicemos, estimulemos, impulsemos, lideremos, un debate profundo, que empiece por la revisión de los programas, de las metodologías, de los planteamientos, de los objetivos, etc. y que nos debe llevar a una presencia más activa en la sociedad, a generar debates, a cuestionar los absolutos, a criticar las soluciones que se proponen a la luz de lo que el pasado nos enseña, en definitiva, a propiciar un conocimiento plural y crítico, que cuestione nuestros propios hallazgos, nuestras propias exposiciones. Que genere escepticismo en el sentido de proponer nuevos caminos, nuevas en rutas. En palabras de N. Chomsky (2014) nuestro papel como educadores, «no consiste en instilar información en la cabeza de alguien que luego la recitará, sino que consiste en capacitar a la gente para que lleguen a ser personas creativas e independientes y puedan encontrar gusto en el descubrimiento, la creación y la creatividad a cualquier nivel o en cualesquiera dominios a los que les lleven sus intereses»?.

La historia, la historia de las relaciones laborales en este caso, tiene un potencial explicativo con el que muy pocas disciplinas puedes contar. Y si además analizamos el conjunto de las materias que formar el grado que nos ocupa $y$, si se diera la coordinación que venimos reclamando hace ya bastante tiempo, estaríamos muy cerca de alcanzar unos resultados realmente interesantes para nuestros alumnos y para nosotros mismos.

Y habría que analizar también la vinculación de las llamadas competencias transversales presentes en todos los grados con las posibilidades que para su desarrollo ofrece materias como la nuestra. A su vez, habría que enlazar esta cuestión con los conocimientos, competencias y habilidades que demandan los empleadores a los demandantes de empleo. Si conseguimos interiorizar este triángulo y fuésemos capaces de trasladarlo a las aulas, estaríamos muy cerca de

9 En este mismo documento:» Un físico mundialmente célebre cuando, en sus cursos para primero de carrera, se le preguntaba "¿qué parte del programa cubriremos este semestre?», contestaba: «no importa lo que cubramos, lo que importa es lo que descubráis vosotros». Tenéis que ganar la capacidad y la autoconfianza en esta asignatura para desafiar y crear e innovar, y así aprenderéis; así haréis vuestro el material y seguir adelante. No es cosa de acumular una serie fijada de hechos que luego podáis soltar por escrito en un examen para olvidarlos al día siguiente». 
conseguir lo que demandan los alumnos de económicas: profesionales capaces de aportar soluciones imaginativas para las dificultades que nos acucian. Pero esa será materia de la siguiente reflexión.

\section{Conclusiones}

Una vez analizado el proceso, las áreas responsables de su impartición y los contenidos de los mismos podemos concluir:

— Los programas analizados de Historia que se imparten en el grado de Relaciones laborales y recursos humanos, presentan un alto grado de homogeneidad, debido a su adscripción significativa al área de Historia Económica y urge una adaptación a la materia específica de Historia de las Relaciones laborales.

- La impartición de la asignatura por áreas de conocimientos distintas no debe llevar a un alejamiento particular de algunas de ellas de los descriptores recogidos en el Libro Blanco de la titulación, como se observa en la tabla 4, donde la variedad de tópicos evidencia que no se siguen dichas pautas. Debemos hacer un esfuerzo por armonizar los contenidos.

- Resulta necesario, a nuestro juicio, la inclusión, de temas actuales (crisis económica, reforma laboral, crisis de las organizaciones sindicales, responsabilidad social corporativa, políticas de empleo y sistemas de colocación, economía social, culturas del trabajo, etc.). Esto nos ayudará a participar en el debate social del momento y del que hemos estado completamente ausentes, tanto docentes como discentes hasta el momento. Introducir a los alumnos en esta dinámica forma parte también del propio proceso educativo, le ayuda a desarrollar las competencias propias del título y, el último lugar, a anticiparse al mundo que se encontrará mańana. Que este debe ser uno de los objetivos fundamentales de la educación.

- Es necesario profundizar en la aportación de nuestras materias a la consecución de las competencias transversales que persigue el grado que nos ocupa.

\section{Referencias bibliográficas}

Сномsкy, A. (2014). «Sobre el trabajo académico, el asalto neoliberal a las universidades y como debería ser la educación superior». Disponible en http://www.sinpermiso.info/ textos/index.php?id=6738

Correa Ramírez, J.J. (2011). «Los giros en la historia: función social de la historia y posmodernidad, un debate que no cesa». Historelo. Revista de historia regional y local. Vol. III, N.o 6. Julio / Diciembre de 2011, pp. 13-38. 
Domínguez Martín, R. (2008). «La Responsabilidad Social Global Empresarial (RSGE): el sector privado y la lucha contra la pobreza». Revista del Ministerio de Trabajo e Inmigración, n. ${ }^{\circ}$ 76, pp. 59-93.

Galán García, A. y Herraiz Martín, M.S. (2005). «La enseñanza de las Relaciones Laborales en Espańa», Trabajo. Revista de la Asociación Estatal de Centros Universitarios y Relaciones Laborales, 16, pp. 17-31.

Galán García, A. (2011). "El proceso de convergencia en al Grado de Relaciones laborales y Recursos humanos. Una ojeada al panorama nacional». En EEES y cambios en las metodologías docentes. Reflexiones y experiencias aplicadas a las Ciencias del trabajo. Valencia: Tirant lo Blanc, 15-45.

Galán García, A. y Manzanares Martínez, D. (2010). «El área de Historia e Instituciones Económicas en el Grado de Relaciones Laborales y Recursos Humanos: orientaciones, contenidos y competencias». IX Encuentro de Didáctica de la Historia Económica. Disponible en http://www.aehe.net/docencia-seccion/pdfs_ congresos/2010toledo/sesion2a/2/galan.pdf

Galán García, A. (2012). «Responsabilidad Social Corporativa: apuntes históricos». En Castellano Burguillo, E. y Rodríguez Piñero-Royo, M.: Estudios sobre Derecho y RSC en un contexto de crisis. Cinca, 2012, pp. 11-29.

Gimbert, X. (2011). Pensar estratégicamente. Modelos, conceptos y reflexiones. Deusto, pp. 41-61.

Iglesias de Ussel, J., Miguel, J.J. de y Trinidad, A. (2009). Sistemas educativos y politicas de educación superior. Consejo Económico y Social.

Новsваwм, E. (1998): Crónica del siglo XX. Crítica.

Judt, T. y Snyder, T. (2012). Pensar el siglo XX. Taurus, 275.

Lerma Montero, I. (coord.) (2005). Libro Blanco del Titulo de Grado en Ciencias Laborales y Recursos Humanos, Madrid: ANECA. Disponible en http://www.aneca.es/var/ media/150308/libroblanco_rrhh_def.pdf

Llopis Angelán, E. (1991). «La función de la Historia Económica en la formación de los economistas», I Encuentro sobre Didáctica de la "Historia Económica», Instituto de Ciencias de la Educación de la Universidad de Zaragoza: Zaragoza, 27-36. Disponible en www.aehe.net/docencia-seccion/pdfs_congresos/temas/.../llopis.pdf

Noiville, F. (2011). Soy economista y os pido disculpas. Deusto.

Román Cervantes, R. (2007). «La historia económica de las relaciones laborales en la universidad de La Laguna: ¿una optativa atractiva?». VIII Encuentro de Didáctica de la Historia económica. La Laguna, 20-21 de septiembre de 2007. Disponible en http:// www.aehe.net/docencia-seccion/pdfs_congresos/temas/propuestas-didacticas/roman. pdf 